\section{Fatores dietéticos e câncer oral: estudo caso-controle na Região Metropolitana de São Paulo, Brasil}

\author{
Dietary factors and oral cancer: \\ a case-control study in Greater \\ Metropolitan São Paulo, Brazil
}

Dirce Maria Lobo Marchioni 1

Regina Mara Fisberg 1

José Francisco de Góis Filho 2

Luiz Paulo Kowalski 2

Marcos Brasilino de Carvalho 3

Márcio Abrahão 4

Maria do Rosario Dias de Oliveira Latorre 1

José Eluf Neto ${ }^{1}$

Victor Wünsch-Filho 1

\footnotetext{
${ }^{1}$ Faculdade de Saúde Pública, Universidade de São Paulo, São Paulo, Brasil. 2 Departamento de Cabeça e Pescoço, Instituto do Câncer Arnaldo Vieira de Carvalho, São Paulo, Brasil.

3 Departamento de Cabeça e Pescoço, Hospital Heliópolis, São Paulo, Brasil.

4 Hospital São Paulo, Universidade Federal de São Paulo, São Paulo, Brasil.

Correspondência D. M. L. Marchioni Departamento de Nutrição, Faculdade de Saúde Pública Universidade de São Paulo. Av. Dr. Arnaldo 715, São Paulo, SP 01246-904, Brasil. marchioni@usp.br
}

\begin{abstract}
Dietary factors associated with oral cancer were investigated in a case-control hospital-based study in the city of São Paulo, Brazil, from 1998 to 2002. A total of 835 subjects, 366 with histologically confirmed incident cases of oral-cavity or pharyngeal cancer and 469 controls participated in the study. Dietary data were collected with a food frequency questionnaire (FFQ). Odds ratios (OR) and 95\% confidence intervals (95\%CI) were obtained from unconditional logistic regression, adjusted for potential confounders. Inverse associations with risk of oral cancer were found for the highest intake of beans, $O R=0.37$ (95\%CI: 0.22-0.64), raw vegetables, $O R=0.51$ (95\%CI: 0.29-0.93) and the intermediate tertile of rice and pasta intake, OR $=0.56$ (95\%CI: 0.380.83). Positive associations were observed for the highest intake of eggs, OR = 1.84 (95\%CI: 1.232.75 ), potatoes, $O R=2.22$ (95\%CI: $1.53-3.25)$, and milk, OR= 1.80 (95\%CI: 1.09-2.98). Some traditional Brazilian foods like rice and beans emerged as protective factors against oral cancer, independently of other risk factors.
\end{abstract}

Mouth Neoplasms; Diet; Case-Control Studies

\section{Introdução}

A cidade de São Paulo apresenta a mais elevada incidência de câncer oral no Brasil e na América Latina 1 . Depois de vários anos de declínio, as taxas de incidência e mortalidade têm aumentado em muitas localidades 2. Em São Paulo, relata-se no período de 1969 a 1998 uma variação positiva de $30 \%$ na incidência em homens e de $179 \%$ em mulheres, apesar da mortalidade no período manter-se estacionada 1 . No Brasil, as estimativas para 2006 são que o câncer de cavidade oral represente $2,8 \%$ de todas as neoplasias malignas, ocupando a sétima posição entre os cânceres mais comuns, e a quinta entre os homens ${ }^{3}$. No mundo, o câncer de cavidade oral e faringe é a quinta incidência de câncer e a sétima causa de morte 4

O câncer oral afeta principalmente os homens, com uma relação de gênero em torno de 10:1 5. As diferentes taxas de incidência que ocorrem em distintas regiões permitem supor que fatores ambientais influenciam os padrões de ocorrência da doença. Os fatores de risco reconhecidos em todo o mundo para o câncer oral são o fumo e álcool 5,6,7. Em contrapartida, evidências científicas apontam para o papel protetor de certos elementos da dieta sobre o câncer oral 5,7. Tendo em vista o papel potencial da alimentação na proteção ou promoção do desenvolvimento de neoplasias malignas, este estudo objetivou investigar os fatores dietéticos associados com o câncer oral. 


\section{Metodologia}

Este estudo analisa os dados no Estudo Internacional de Fatores Ambientais, Vírus e Câncer da Cavidade Oral e da Laringe, um estudo multicêntrico, do tipo caso-controle de base hospitalar, coordenado pela International Agency for Research on Cancer (IARC). Além de São Paulo, participaram outras quatro cidades brasileiras e duas em países latinos. O presente estudo utiliza os dados coletados em São Paulo. O estudo multicêntrico foi aprovado pela Comissão Nacional de Ética em Pesquisa (CONEP), e este estudo foi aprovado pelo Comitê de Ética da Faculdade de Saúde Pública, Universidade de São Paulo.

\section{População de estudo}

Foram inicialmente convidados a participar do estudo 1.097 indivíduos. Destes, 41 recusaram-se a participar e 12 apresentaram condições físicas ou mentais que impossibilitaram a realização da entrevista. Quanto ao status no estudo, 607 eram potencialmente casos, ou seja, sem confirmação histológica e 490 controles. Foram incluídos os casos de câncer classificados com os códigos C00 a C14 (cavidade oral e orofaringe) de acordo com a Classificação Internacional de Doenças (CID10) 8 , com exceção dos casos classificados como C00.0, C00.1 e C00.2, relativos ao lábio externo e C11 (nasofaringe). A exclusão destes últimos baseou-se na literatura, que postula que os cânceres nesses sítios não compartilham os mesmos fatores de risco. Foi também excluído um indivíduo que não forneceu dados de consumo dietético. Dessa forma, a amostra final foi de 835 indivíduos, sendo 366 casos com confirmação histológica e 469 controles, recrutados entre novembro de 1998 a março de 2002. Dos indivíduos não participantes do estudo, 43 eram homens e 10 mulheres. Tanto para os potencialmente casos quanto para os potencialmente controles, não houve diferença estatisticamente significativa entre participantes e não participantes com relação à idade (respectivamente, $\mathrm{p}=0,311 \mathrm{e} \mathrm{p}=$ $0,468)$ e distribuição quanto a sexo ( $\mathrm{p}=0,148$ e $\mathrm{p}$ $=0,258$, respectivamente) .

Os controles foram selecionados entre os pacientes admitidos nos hospitais gerais participantes do estudo, por condições não relacionadas com doenças associadas aos fatores de risco para câncer de cavidade oral e faringe: doenças dos aparelhos digestório (24\%), circulatório (21\%), sistema genito-urinário $(9 \%)$, causa externas $(9 \%)$, entre outras. Os controles foram pareados aos casos segundo sexo e idade em qüinqüênios, pela distribuição esperada dos casos (pareamento por freqüência). Os controles não poderiam ter história ou suspeita de câncer de cavidade oral ou de laringe no presente ou passado.

\section{Coleta de dados}

O questionário foi desenvolvido para o estudo multicêntrico e incluiu indicadores sócio-culturais, uma detalhada história ocupacional, condições de moradia, tabagismo, consumo de álcool e mate, história familiar de câncer, antecedentes de infecções selecionadas e um inquérito dietético. Os casos foram identificados e entrevistados antes do início do tratamento específico da doença. O presente estudo utilizou os dados obtidos no inquérito dietético, dados sócio-demográficos, tabagismo e consumo de bebidas alcoólicas e relato de peso corporal dois anos antes da entrevista.

\section{Inquérito dietético}

O inquérito alimentar foi realizado por meio de um Questionário de Freqüência Alimentar (QFA) semiquantitativo desenvolvido pela IARC. A lista de alimentos do QFA é composta por 27 alimentos, grupos de alimentos ou preparações. Para cada item, foi estabelecida a porção usual de consumo. Cada participante do estudo foi solicitado a responder, para cada item do QFA, com que média de freqüência semanal aquela quantidade de alimento era consumida, antes dos sintomas da doença surgirem. As respostas foram abertas, o que permitiu o tratamento dessa variável como contínua. Foi investigada a freqüência de consumo (porções por semana) dos seguintes alimentos: leite, iogurte, manteiga, pão, macarrão ou arroz, pratos à base de milho (ex. polenta, creme de milho), mandioca, farinha de mandioca, carne bovina, carne suína, frango, peixe, embutidos, ovos, queijos, batata, vegetais crus e saladas, crucíferas, cenoura, tomate, feijão e leguminosas, suco de frutas, maçã ou pêra, frutas cítricas, banana, bolos e doces. Em segui$\mathrm{da}$, foram feitos agrupamentos tendo por base o conteúdo nutricional ou botânico assemelhado dos itens do QFA. Inicialmente, os grupamentos foram: cereais e derivados (pães, arroz e massas e milho); raízes e tubérculos (mandioca, farinha de mandioca e batata); laticínios (leite, iogurte e queijo); carne vermelha (carne bovina e suína); carne branca (carne de ave e peixe); vegetais (salada crua, crucíferas, cenoura e tomate) e frutas (sucos de frutas, maçãs, banana e cítricos). A seguir, novo rearranjo foi feito, no sentido de obter menor número de grupos, e, portanto, uma medida mais resumida da dieta: carnes (carne vermelha, carne branca e embutidos); vegetais 
e frutas; alimentos básicos (cereais, raízes e tubérculos e leguminosas); e alimentos de origem animal (laticínios, ovos e carne total). A somatória das freqüências de consumo dos alimentos de cada grupo discriminou o consumo. Essas variáveis foram categorizadas em tercis de freqüência de consumo de acordo com a distribuição da população de controles.

\section{Co-variáveis}

Foram obtidos dados demográficos, sócio-culturais e de hábitos de vida, bem como relato do peso corporal dois anos antes da entrevista.

$\mathrm{O}$ tabagismo foi mensurado levando-se em conta a experiência de fumo durante todo o período de vida, definido como a exposição cumulativa para o número de pacotes de cigarros consumidos diariamente: tabaco em maços $v s$. ano ( $p a-$ ck years). Foi considerado o consumo de cigarro, charuto e cachimbo ${ }^{9}$. Estabeleceu-se o consumo médio diário de pacotes de cigarros e multiplicou-se pelo número de anos como fumante.

Para o etilismo, considerou-se o consumo médio de álcool em gramas por dia. Assim, inicialmente transformou-se o consumo de bebidas relatado pelo indivíduo em gramas de álcool 10.

\section{Análise estatística}

Para a análise multivariada foram utilizadas para o ajuste as variáveis de pareamento do estudo, sexo e idade e as variáveis já reconhecidas como fatores de risco: tabagismo e etilismo. Na modelagem estatística selecionaram-se as variáveis cujos valores de p (nível de significância descritivo do teste) foram menores ou iguais a 0,20 na análise bruta. A seguir, essas variáveis foram introduzidas no modelo de regressão logística por meio do procedimento stepwise. Foram mantidas no modelo as variáveis que permaneceram significativas depois do ajuste pelas outras variáveis. No entanto, apesar da variável escolaridade não ter alterado nenhuma associação, optou-se por mantê-la no modelo, pois esta variável pode ser considerada um indicador de status social e econômico, e, portanto, importante fator na seleção dos alimentos para consumo. Assim, as estimativas de risco associadas às variáveis dietéticas estão ajustadas por sexo, idade, consumo de tabaco, consumo de álcool, peso relatado pelo indivíduo dois anos antes da entrevista, somatória do número de porções dos itens alimentares consumidos, macrorregião de nascimento e escolaridade.

Foram testadas as interações (modificação de efeito) incluindo-se termos multiplicativos entre as variáveis do modelo e verificando-se seu sig- nificado estatístico. Não foram observadas interações entre elas.

O efeito dose-resposta foi testado pelo teste $\chi^{2}$ para tendência linear, ajustado para o sexo, idade, tabagismo e etilismo.

Todas as análises foram feitas no programa estatístico Intercoled STATA for Windows 98/95/ NT (Stata Corporation, College Station, Estados Unidos).

\section{Resultados}

As características demográficas e sociais dos participantes do estudo estão descritas na Tabela 1. A média de idade dos casos foi de 55,5 (DP = $12,4)$ anos e a dos controles foi de 56,5 (DP $=10,9)$ anos.

Observou-se maior proporção de fumantes entre os casos em relação aos controles, como era esperado. Da mesma forma, observou-se que tanto a proporção de indivíduos que relatam consumir bebidas alcoólicas correntemente quanto o consumo médio de álcool por dia é significativamente maior entre casos que entre controles.

O consumo de leite, iogurte e manteiga (Tabela 2) foi fator protetor na análise univariada. Após o ajuste, o consumo de manteiga se manteve como fator de proteção, porém, observou-se que o mais alto tercil de consumo de leite, correspondente a mais que sete porções semanais, associou-se positivamente ao câncer oral.

O consumo de 14 porções por semana de arroz e massas, correspondente ao segundo tercil, mostrou-se como fator de proteção (OR ajusta$\mathrm{da}=0,56$, IC95\%: 0,38-0,83). Por outro lado, o consumo de batatas associou-se positivamente com o câncer oral, além deste alimento também ter apresentado tendência significativa para aumento de risco de acordo com o aumento do consumo.

Não se observou associação entre o consumo de carne, quer bovina ou de outro animal, e o câncer oral. No entanto, o consumo de ovos apresentou-se como fator de risco, mesmo após o ajuste da estimativa pelos fatores de controle, apresentando ainda tendência de aumento de risco com o aumento dos níveis de exposição significativa estatisticamente (Tabela 2 ).

O consumo de feijão e de vegetais crus associaram-se negativamente ao câncer oral e apresentaram tendência significativa para maior proteção quanto maior o consumo. O consumo de cereais no tercil intermediário, entre 17 a 22 porções por semana, mostrou uma associação negativa, estatisticamente significativa, para o câncer oral (Tabela 2). 
Tabela 1

Distribuição do número e porcentagem de casos e controles, segundo características sociais e demográficas. São Paulo, Brasil, $1998-2002$.

\begin{tabular}{|c|c|c|c|c|c|}
\hline \multirow[t]{2}{*}{ Variável } & \multicolumn{2}{|c|}{ Casos $(n=366)$} & \multicolumn{2}{|c|}{ Controles $(n=469)$} & \multirow[t]{2}{*}{ Valor $p\left(\chi^{2}\right)$} \\
\hline & $\mathbf{n}$ & $\%$ & $\mathrm{n}$ & $\%$ & \\
\hline \multicolumn{6}{|l|}{ Sexo } \\
\hline Homem & 310 & 84,7 & 370 & 78,9 & * \\
\hline Mulher & 56 & 15,3 & 99 & 21,1 & \\
\hline \multicolumn{6}{|l|}{ Idade (anos) } \\
\hline$<40$ & 19 & 5,2 & 40 & 8,5 & * \\
\hline 40 -50 & 93 & 25,4 & 102 & 21,7 & \\
\hline 50 -60 & 130 & 35,5 & 135 & 28,8 & \\
\hline $60+70$ & 84 & 23,0 & 118 & 25,2 & \\
\hline$\geq 70$ & 40 & 10,9 & 74 & 15,8 & \\
\hline \multicolumn{6}{|l|}{ Escolaridade } \\
\hline Analfabeto & 47 & 12,8 & 65 & 13,9 & 0,200 \\
\hline Quartas primeiras séries do primeiro grau (antigo primário) & 202 & 55,2 & 225 & 48,2 & \\
\hline Primeiro grau completo & 64 & 17,5 & 93 & 19,9 & \\
\hline Segundo grau & 42 & 11,5 & 57 & 12,2 & \\
\hline Universitário & 11 & 3,0 & 27 & 5,8 & \\
\hline \multicolumn{6}{|l|}{ Região de nascimento } \\
\hline Norte & 1 & 0,3 & 1 & 0,3 & 0,21 \\
\hline Nordeste & 95 & 26,2 & 161 & 26,2 & \\
\hline Centro-oeste & 2 & 0,6 & 2 & 0,6 & \\
\hline Sudeste & 242 & 66,8 & 274 & 66,8 & \\
\hline Sul & 13 & 3,6 & 14 & 3,6 & \\
\hline Estrangeiro & 9 & 2,5 & 10 & 2,5 & \\
\hline \multicolumn{6}{|l|}{ Consumo de tabaco (maço vs. ano) } \\
\hline 아-1 & 142 & 31,2 & 20 & 5,6 & $<0,001$ \\
\hline $1 \mid-25$ & 140 & 30,8 & 100 & 27,9 & \\
\hline $25+50$ & 118 & 25,9 & 142 & 39,7 & \\
\hline$\geq 50$ & 55 & 12,1 & 96 & 26,8 & \\
\hline \multicolumn{6}{|l|}{ Período como fumante (anos) } \\
\hline 아-25 & 219 & 48,1 & 64 & 17,9 & $<0,001$ \\
\hline $25+35$ & 90 & 19,8 & 104 & 29,0 & \\
\hline$\geq 35$ & 144 & 32,1 & 190 & 53,1 & \\
\hline \multicolumn{6}{|l|}{ Bebida alcoólica } \\
\hline Nunca bebeu & 142 & 30,3 & 26 & 7,1 & $<0,001$ \\
\hline Bebeu no passado & 180 & 38.5 & 151 & 41,0 & \\
\hline Bebe correntemente & 146 & 31,2 & 190 & 51,9 & \\
\hline \multicolumn{6}{|l|}{ Consumo alcoólico médio (g/d) } \\
\hline Não bebe & 142 & 30.9 & 26 & 7,1 & $<0,001$ \\
\hline $0,01-40$ & 174 & 37,9 & 112 & 30,8 & \\
\hline 40 -80 & 62 & 13,5 & 70 & 19,2 & \\
\hline $80+-120$ & 27 & 5,9 & 46 & 12,7 & \\
\hline$\geq 120$ & 54 & 11,7 & 110 & 30,2 & \\
\hline
\end{tabular}

* Variáveis de pareamento do estudo. 
Valores de odds ratio (OR) e intervalo com 95\% de confiança (IC95\%) para câncer oral obtidos por regressão logística não condicional, univariada e múltipla segundo tercis aproximados dos itens alimentares. São Paulo, Brasil, 1998-2002.

\begin{tabular}{|c|c|c|c|c|c|}
\hline Alimento & $\begin{array}{c}\text { Tercis } \\
\text { (porções/semana) }\end{array}$ & $\begin{array}{l}\text { Controle: } \\
\text { Caso }\end{array}$ & $\begin{array}{c}\mathrm{OR} * \\
\text { (IC95\%) }\end{array}$ & $\begin{array}{l}\text { OR ** } \\
\text { (IC95\%) }\end{array}$ & 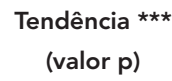 \\
\hline \multirow[t]{3}{*}{ Leite } & $\leq 3$ & $164: 157$ & 1,00 & 1,00 & 0,551 \\
\hline & $3+7$ & 209:121 & $0,60(0,44-0,83)$ & $0,79(0,54-1,16)$ & \\
\hline & $>7$ & 109:88 & $0,84(0,59-1,20)$ & $1,80(1,09-2,98)$ & \\
\hline \multirow[t]{2}{*}{ logurte } & $<1$ & $365: 299$ & 1,00 & 1,00 & 0,223 \\
\hline & $\geq 1$ & $116: 67$ & $0,70(0,50-0,99)$ & $0,83(0,54-1,28)$ & \\
\hline \multirow[t]{3}{*}{ Manteiga } & $\leq 3$ & $178: 187$ & 1,00 & 1,00 & 0.001 \\
\hline & $3+7$ & $234: 136$ & $0,55(0,41-0,74)$ & $0,57(0,39-0,84)$ & \\
\hline & $>7$ & $70: 43$ & $0,58(0,38-0,90)$ & $0,63(0,35-1,11)$ & \\
\hline \multirow[t]{3}{*}{ Queijo } & $\leq 0$ & $191: 163$ & 1,00 & 1,00 & 0,977 \\
\hline & 아-2 & $153: 104$ & $0,80(0,57-1,10)$ & $0,87(0,58-1,30)$ & \\
\hline & $>2$ & $138: 98$ & $0,83(0,60-1,16)$ & $1,12(0,74-1,73)$ & \\
\hline \multirow[t]{3}{*}{ Pão } & $<7$ & $121: 135$ & 1,00 & 1,00 & 0,407 \\
\hline & 7 & 249:145 & $0,52(0,38-0,72)$ & $0,69(0,45-1,05)$ & \\
\hline & $>7$ & $112: 86$ & $0,69(0,47-1,00)$ & $0,72(0,42-1,22)$ & \\
\hline \multirow[t]{3}{*}{ Arroz e massas } & $<14$ & $150: 126$ & 1,00 & 1,00 & 0,522 \\
\hline & $=14$ & $306: 209$ & $0,81(0,61-1,09)$ & $0,56(0,38-0,83)$ & \\
\hline & $>14$ & $26: 30$ & $1,38(0,77-2,44)$ & $1,15(0,54-2,45)$ & \\
\hline \multirow[t]{3}{*}{ Pratos à base de milho } & $<1$ & $284: 198$ & 1,00 & 1,00 & 0,293 \\
\hline & $=1$ & $109: 84$ & $1,10(0,79-1,55)$ & $1,12(0,73-1,71)$ & \\
\hline & $>1$ & $88: 83$ & $1,34(0,94-1,90)$ & $1,12(0,72-1,73)$ & \\
\hline \multirow[t]{3}{*}{ Mandioca } & $<1$ & $299: 232$ & 1,00 & 1,00 & 0,804 \\
\hline & $=1$ & $114: 75$ & $0,85(0,60-1,19)$ & $0,91(0,60-1,38)$ & \\
\hline & $>1$ & $65: 56$ & $1,11(0,75-1,65)$ & $1,26(0,77-2,07)$ & \\
\hline \multirow[t]{3}{*}{ Farinha de mandioca } & $<1$ & $265: 203$ & 1,00 & 1,00 & 0,805 \\
\hline & $=1$ & $81: 52$ & $0,84(0,56-1,24)$ & $0,82(0,50-1,36)$ & \\
\hline & $>1$ & $130: 108$ & $1,08(0,79-1,48)$ & $1,01(0,67-1,52)$ & \\
\hline \multirow[t]{3}{*}{ Batata } & $\leq 1$ & $200: 99$ & 1,00 & 1,00 & $<0,001$ \\
\hline & $1+3$ & $195: 170$ & $1,76(1,28-2,41)$ & $1,91(1,30-2,81)$ & \\
\hline & $>3$ & $87: 96$ & $2,22(1,53-3,25)$ & $2,46(1,53-3,95)$ & \\
\hline \multirow[t]{3}{*}{ Doces ou bolos } & $\leq 0$ & $184: 157$ & 1,00 & 1,00 & 0,602 \\
\hline & 아-2 & $61: 45$ & $0,86(0,56-1,34)$ & $1,05(0,61-1,85)$ & \\
\hline & $>2$ & $136: 94$ & $0,81(0,57-1,13)$ & $0,94(0,61-1,43)$ & \\
\hline \multirow[t]{3}{*}{ Carne bovina } & $\leq 3$ & $257: 193$ & 1,00 & 1,00 & 0,296 \\
\hline & $3+5$ & $80: 58$ & $0,96(0,66-1,42)$ & $0,85(0,53-1,36)$ & \\
\hline & $>5$ & $145: 113$ & $1,04(0,76-1,41)$ & $0,97(0,64-1,45)$ & \\
\hline \multirow[t]{2}{*}{ Carne suína } & $<1$ & $368: 253$ & 1,00 & 1,00 & 0,287 \\
\hline & $\geq 1$ & $114: 113$ & $1,45(1,06-1,95)$ & $1,13(0,78-1,64)$ & \\
\hline \multirow[t]{3}{*}{ Frango ou galinha } & $\leq 2$ & $274: 226$ & 1,00 & 1,00 & 0,262 \\
\hline & $2+3$ & $74: 68$ & $1,12(0,77-1,62)$ & $1,06(0,67-1,67)$ & \\
\hline & $>3$ & $134: 72$ & $0,65(0,47-0,91)$ & $0,70(0,46-1,07)$ & \\
\hline \multirow[t]{3}{*}{ Peixe } & $<1$ & $271: 213$ & 1,00 & 1,00 & 0,680 \\
\hline & $=1$ & $141: 105$ & $0,95(0,70-1,30)$ & $0,90(0,61-1,33)$ & \\
\hline & $>1$ & $69: 48$ & $0,89(0,59-1,33)$ & $1,17(0,70-1,96)$ & \\
\hline \multirow{3}{*}{ Embutidos } & $<1$ & 248:177 & 1,00 & 1,00 & 0,931 \\
\hline & $=1$ & $128: 83$ & $0,91(0,65-1,28)$ & $0,77(0,50-1,19)$ & \\
\hline & $>1$ & $105: 106$ & $1,42(1,02-1,98)$ & $1,49(0,99-2,27)$ & \\
\hline
\end{tabular}

(continua) 
Tabela 2 (continuação)

\begin{tabular}{|c|c|c|c|c|c|}
\hline Alimento & $\begin{array}{c}\text { Tercis } \\
\text { (porções/semana) }\end{array}$ & $\begin{array}{c}\text { Controle: } \\
\text { Caso }\end{array}$ & $\begin{array}{c}\text { OR * } \\
(\text { IC95\%) }\end{array}$ & $\begin{array}{l}\text { OR ** } \\
(\text { IC95\%) }\end{array}$ & $\begin{array}{c}\text { Tendência *** } \\
\text { (valor } \mathrm{p} \text { ) }\end{array}$ \\
\hline \multirow[t]{3}{*}{ Ovos } & $\leq 1$ & 275:155 & 1,00 & 1,00 & 0,005 \\
\hline & 1 -2 & $73: 69$ & $1,68(1,14-2,46)$ & $2,06(1,29-3,31)$ & \\
\hline & $>2$ & $134: 140$ & $1,85(1,36-2,52)$ & $1,84(1,23-2,75)$ & \\
\hline \multirow[t]{3}{*}{ Vegetais crus } & $\leq 3$ & $184: 188$ & 1,00 & 1,00 & 0,001 \\
\hline & $3+7$ & $227: 145$ & $0,62(0,46-0,84)$ & $0,58(0,40-0,83)$ & \\
\hline & $>7$ & $71: 33$ & $0,45(0,29-0,72)$ & $0,51(0,29-0,93)$ & \\
\hline \multirow[t]{2}{*}{ Crucíferas } & $<1$ & $169: 115$ & 1,00 & 1,00 & 0,393 \\
\hline & $\geq 1$ & $312: 362$ & $1,17(0,88-1,57)$ & $1,25(0,88-1,79)$ & \\
\hline \multirow[t]{3}{*}{ Cenoura } & $\leq 1$ & $289: 230$ & 1,00 & 1,00 & 0,667 \\
\hline & 1 -2 & $75: 46$ & $0,77(0,51-1,15)$ & $0,92(0,56-1,51)$ & \\
\hline & $>2$ & 118:88 & $0,93(0,67-1,30)$ & $1,17(0,77-1,79)$ & \\
\hline \multirow[t]{3}{*}{ Tomate } & $\leq 1$ & 190:141 & 1,00 & 1,00 & 0,489 \\
\hline & 1 -3 & $134: 115$ & $1,15(0,82-1,60)$ & $1,34(0,89-2,04)$ & \\
\hline & $>3$ & $158: 109$ & $0,93(0,67-1,29)$ & $0,99(0,64-1,52)$ & \\
\hline \multirow[t]{3}{*}{ Feijão } & $\leq 7$ & $72: 63$ & 1,00 & 1,00 & 0,008 \\
\hline & 7ト-14 & $157: 141$ & $1,03(0,68-1,54)$ & $0,56(0,33-0,97)$ & \\
\hline & $>14$ & $253: 160$ & $0,72(0,49-1,07)$ & $0,37(0,22-0,64)$ & \\
\hline \multirow[t]{3}{*}{ Suco de frutas } & $<1$ & $178: 167$ & 1,00 & 1,00 & 0,371 \\
\hline & $1+5$ & $177: 114$ & $0,69(0,50-0,95)$ & $0,84(0,57-1,24)$ & \\
\hline & $>5$ & $127: 84$ & $0,70(0,50-1,00)$ & $1,07(0,68-1,70)$ & \\
\hline \multirow[t]{3}{*}{ Maçãs } & $\leq 0$ & 213:199 & 1,00 & 1,00 & 0,089 \\
\hline & 아-1 & $117: 67$ & $0,61(0,43-0,87)$ & $0,86(0,55-1,34)$ & \\
\hline & $>1$ & $152: 98$ & $0,69(0,50-0,95)$ & $1,17(0,78-1,77)$ & \\
\hline \multirow[t]{3}{*}{ Frutas cítricas } & $\leq 2$ & 214:190 & 1,00 & 1,00 & 0,182 \\
\hline & $2 \mid-5$ & $109: 73$ & $0,75(0,53-1,07)$ & $0,88(0,56-1,39)$ & \\
\hline & $>5$ & $159: 101$ & $0,71(0,52-0,98)$ & $0,85(0,55-1,32)$ & \\
\hline \multirow[t]{3}{*}{ Bananas } & $\leq 2$ & $225: 183$ & 1,00 & 1,00 & 0,412 \\
\hline & $2 \mid-5$ & $114: 93$ & $1,00(0,71-1,40)$ & $1,15(0,75-1,76)$ & \\
\hline & $>5$ & $143: 88$ & $0,76(0,54-1,05)$ & $0,96(0,62-1,47)$ & \\
\hline
\end{tabular}

* Univariada;

** Modelo ajustado por sexo, idade, consumo de tabaco, álcool, educação, número de porções, peso, região de nascimento;

*** Mantel Hanzel qui-quadrado para tendência, ajustado por sexo, idade, consumo de álcool e tabaco.

Para o consumo de laticínios, verificou-se efeito protetor para o consumo no tercil intermediário, ou seja, de 7 a 11 porções semanais. Porém, o consumo mais elevado, maior que 11 porções/semana, passou a ter o efeito inverso. No entanto, essas associações não apresentaram significado estatístico (Tabela 3). Para o consumo de carnes (Tabela 3), quer carne vermelha, branca ou para o somatório de consumo de todos os tipos, não se observou associação estatisticamente significativa.

Observou-se tendência significativa para diminuição do risco de câncer oral quanto maior o consumo de vegetais e frutas. Quando se agrupam os alimentos de origem vegetal, bem como os alimentos básicos, verifica-se igualmente diminuição do risco, porém sem significado estatístico (Tabela 4).

\section{Discussão}

Este estudo, do tipo caso-controle de base hospitalar, baseado em dados obtidos no Município de São Paulo, buscou verificar as associações entre alimentos e grupos de alimentos e o câncer oral. Foi possível observar que casos de câncer oral diferiram dos controles em determinados aspectos do seu comportamento alimentar: no menor consumo de arroz e massas, feijão e vegetais crus e saladas. Esses achados foram independentes do consumo de álcool, tabagismo e demais variáveis de controle.

Neste estudo, o feijão apresentou-se como fator protetor para o câncer oral, com tendência significativa para diminuição do risco com o aumento do consumo. As leguminosas têm recebido destaque na literatura científica recen- 
Valores de odds ratio (OR) e intervalo com 95\% de confiança (IC95\%) para câncer oral obtidos por regressão logística não condicional, univariada e múltipla segundo tercis aproximados de grupos de alimentos. São Paulo, Brasil, 1998-2002.

\begin{tabular}{|c|c|c|c|c|c|}
\hline $\begin{array}{l}\text { Grupos de } \\
\text { alimentos }\end{array}$ & $\begin{array}{c}\text { Tercis } \\
\text { (porções/semana) }\end{array}$ & $\begin{array}{l}\text { Controle: } \\
\text { Caso }\end{array}$ & $\begin{array}{c}\text { OR * } \\
\text { (IC95\%) }\end{array}$ & $\begin{array}{l}\text { OR ** } \\
\text { (IC95\%) }\end{array}$ & $\begin{array}{l}\text { Tendência *** } \\
\text { (valor } \mathrm{p})\end{array}$ \\
\hline \multirow[t]{3}{*}{ Cereais e derivados } & $\leq 17$ & 160:147 & 1,00 & 1,00 & 0,227 \\
\hline & $17 \mid-22$ & $181: 104$ & $0,62(0,44-0,86)$ & $0,57(0,37-0,87)$ & \\
\hline & $>22$ & 140:114 & $0,88(0,63-1,24)$ & $0,76(0,47-1,23)$ & \\
\hline \multirow[t]{3}{*}{ Raízes e tubérculos } & $\leq 3$ & $212: 141$ & 1,00 & 1,00 & 0,460 \\
\hline & $3+6$ & $124: 98$ & $1,17(0,84-1,65)$ & $1,18(0,77-1,79)$ & \\
\hline & $>6$ & $138: 123$ & $1,34(0,95-1,85)$ & $1,23(0,81-1,87)$ & \\
\hline \multirow[t]{3}{*}{ Laticínios } & $\leq 7$ & $228: 193$ & 1,00 & 1,00 & 0,393 \\
\hline & 가-11 & $101: 64$ & $0,75(0,52-1,08)$ & $0,76(0,48-1,19)$ & \\
\hline & $>11$ & $152: 108$ & $0,84(0,61-1,15)$ & $1,72(1,08-2,68)$ & \\
\hline \multirow[t]{3}{*}{ Carne vermelha } & $\leq 3$ & $216: 163$ & 1,00 & 1,00 & 0,219 \\
\hline & $3+6$ & $124: 87$ & $0,93(0,66-1,35)$ & $0,75(0,49-1,14)$ & \\
\hline & $>6$ & $142: 114$ & $1,06(0,77-1,46)$ & $0,89(0,58-1,35)$ & \\
\hline \multirow[t]{3}{*}{ Carne branca } & $\leq 2$ & $208: 167$ & 1,00 & 1,00 & 0,850 \\
\hline & $2+4$ & $141: 116$ & $1,02(0,74-1,41)$ & $1,04(0,70-1,55)$ & \\
\hline & $>4$ & $132: 82$ & $0,77(0,55-1,08)$ & $0,95(0,62-1,46)$ & \\
\hline \multirow[t]{3}{*}{ Vegetais } & $\leq 8$ & $189: 172$ & 1,00 & 1,00 & 0,043 \\
\hline & 8 -15 & $148: 114$ & $0,85(0,61-1,15)$ & $1,09(0,73-1,66)$ & \\
\hline & $>15$ & $144: 78$ & $0,59(0,42-0,84)$ & $0,73(0,46-1,16)$ & \\
\hline \multirow[t]{3}{*}{ Frutas } & $\leq 7$ & $160: 148$ & 1,00 & 1,00 & 0,089 \\
\hline & 7 ㄴ16 & $163: 123$ & $0,83(0,59-1,13)$ & $1,08(0,71-1,65)$ & \\
\hline & $>16$ & $159: 93$ & $0,63(0,45-0,89)$ & $0,89(0,54-1,47)$ & \\
\hline
\end{tabular}

* Univariada;

** Modelo ajustado por sexo, idade, consumo de tabaco, álcool, educação, número de porções, peso, região de nascimento;

*** Mantel Hanzel qui-quadrado para tendência, ajustado por sexo, idade, consumo de álcool e tabaco.

temente. Atualmente seu consumo vem sendo recomendado por organizações de saúde, pois aparentemente colabora para a redução de riscos das doenças crônicas não transmissíveis ? Esses grãos são uma fonte de fibras dietéticas, amido-resistente, oligossacarídeos e carboidratos fermentáveis. São também ricos em antioxidantes, incluindo minerais traços, compostos fenólicos, além de ácido fítico. Os grãos integrais têm uma digestão e absorção mais lenta do que, por exemplo, alimentos refinados, e diminuem a resposta glicêmica sangüínea pós-prandial. Esses mecanismos, isoladamente ou em conjunto, poderiam explicar o efeito protetor atribuído a esse alimento, nas várias fases do processo carcinogênico 11. No entanto, os resultados dos estudos epidemiológicos são inconsistentes, e a avaliação da possível contribuição quantitativa para a diminuição do risco permanece incerta 7,12. Nos estudos de Kjaerheim et al. 6, Franceschi et al. 13 e Garrote et al. 14, as leguminosas associaram-se positivamente ao câncer, enquanto Petridou et al. 15, Kreimer et al. 16 e De Stefani et al. 17 observaram associação negativa para leguminosas.

Nos estudos de Chyou et al. 18, Garrote et al. 14, De Stefani et al. 17, o consumo mais freqüente de arroz e massas associou-se com aumento do risco para o câncer oral. Porém Franceschi et al. 13, em um dos maiores estudos conduzidos na Europa, não observaram associação, como no estudo prévio. Zheng et al. 19 e Petridou et al. 15 observaram associação inversa, como constatado no presente estudo.

Para o World Cancer Research Fund 7, os resultados dos estudos sobre o consumo de leite ou laticínios e o câncer oral são inconsistentes e não permitem julgamento. A maioria, coorte 18 e caso-controle, 13,14,15,17,20 relatam resultados nulos. No presente estudo, o consumo de mais de 11 porções semanais representou excesso de risco para esse câncer. Tal achado deve ser recebido com cautela, devendo ser melhor investigado. Pode significar uma substituição das refeições estruturadas, em geral mais variadas e 
Valores de odds ratio (OR) e intervalo com 95\% de confiança (IC95\%) para câncer oral obtidos por regressão logística não condicional, univariada e múltipla segundo tercis aproximados de grupos de alimentos. São Paulo, Brasil, 1998-2002.

\begin{tabular}{|c|c|c|c|c|c|}
\hline $\begin{array}{l}\text { Grupos de } \\
\text { alimentos }\end{array}$ & $\begin{array}{c}\text { Tercis } \\
\text { (porções/semana) }\end{array}$ & $\begin{array}{c}\text { Controle: } \\
\text { Caso }\end{array}$ & $\begin{array}{c}\text { OR * } \\
\text { (IC95\%) }\end{array}$ & $\begin{array}{c}\text { OR ** } \\
\text { (IC95\%) }\end{array}$ & $\begin{array}{c}\text { Tendência *** } \\
\text { (valor p) }\end{array}$ \\
\hline \multirow[t]{3}{*}{ Carnes } & $\leq 6$ & $177: 142$ & 1,00 & 1,00 & 0,438 \\
\hline & $6+10$ & $178: 131$ & $0,92(0,67-1,26)$ & $1,01(0,68-1,51)$ & \\
\hline & $>10$ & $123: 91$ & $0,92(0,65-1,31)$ & $0,98(0,62-1,56)$ & \\
\hline \multirow[t]{3}{*}{ Vegetais e frutas } & $\leq 18$ & $175: 172$ & 1,00 & 1,00 & 0,010 \\
\hline & 18 -32 & $154: 110$ & $0,73(0,53-1,00)$ & $0,84(0,55-1,29)$ & \\
\hline & $>32$ & $152: 81$ & $0,54(0,38-0,76)$ & $0,73(0,43-1,26)$ & \\
\hline \multirow[t]{3}{*}{ Alimentos básicos } & $\leq 31$ & $163: 130$ & 1,00 & 1,00 & 0,167 \\
\hline & $31-41$ & 153:119 & $0,97(0,70-1,36)$ & $0,69(0,45-1,05)$ & \\
\hline & $>41$ & $157: 110$ & $0,88(0,63-1,23)$ & $0,74(0,45-1,21)$ & \\
\hline \multirow[t]{3}{*}{ Alimentos de origem animal } & $\leq 16$ & $183: 145$ & 1,00 & 1,00 & 0,680 \\
\hline & $16 \mid-24$ & 119:93 & $0,99(0,69-1,40)$ & $1,17(0,75-1,84)$ & \\
\hline & $>24$ & $174: 125$ & $0,91(0,66-1,24)$ & $1,54(0,94-2,51)$ & \\
\hline
\end{tabular}

* Univariada;

* Modelo ajustado por sexo, idade, consumo de tabaco, álcool, educação, número de porções, peso, região de nascimento;

* Mantel Hanzel qui-quadrado para tendência, ajustado por sexo, idade, consumo de álcool e tabaco.

com maior aporte nutritivo, por bebidas lácteas e lanches, mais monótonas e com maior consumo de embutidos, em geral implicados como fatores de risco 13,16 .

Consistente com os estudos anteriores $14,16,20,21,22$, a categoria de consumo mais elevado para os vegetais crus apresentou aproximadamente metade do risco para o câncer oral. Além dos vegetais crus, as evidências para o efeito protetor dos vegetais são consistentes para cenoura, vegetais verdes e amarelos e tomates 14,19,21, mas fraca para crucíferas, para as quais efeitos nulos ou de risco foram descritos 14 , possivelmente devido às propriedades antioxidantes e, no caso de compostos com atividade de vitamina A como o beta-caroteno, ao papel na diferenciação das células epiteliais 23 . No entanto, não se observou, neste estudo, efeito protetor para o consumo de cenouras e tomates em relação ao câncer oral. Os resultados nulos verificados em estudo para o consumo de cenoura, crucíferas e tomates podem ser devido a vieses como os de atenuação, devido à imprecisão do instrumento ou falta de heterogeneidade na população estudada 24 .

Observou-se ainda que o consumo aumentado de ovos associou-se diretamente com o câncer oral. Apesar desse alimento ter sido investigado em vários estudos, os resultados são inconsistentes. Franceschi et al. 13 e Levi et al. 20 observaram associação positiva e estatisticamente significativa entre ovos e câncer oral, além de tendência significativa para aumento de risco com aumento de consumo. Zheng et al. ${ }^{19} \mathrm{e}$ De Stefani et al. 17 observaram uma associação negativa, enquanto Kjaerheim et al. ${ }^{6}$ e De Stefani et al. 25 observaram associações positivas, porém todas não significativas estatisticamente. É possível que esse efeito seja devido à gordura saturada e colesterol 20 .

Observou-se ainda que o consumo aumentado de batatas associou-se positivamente com câncer oral. Um número menor de estudos relatou resultados para esse alimento. Levi et al. $20 \mathrm{e}$ De Stefani et al. 25 verificaram associações positivas, porém não significativas, enquanto Franceschi et al. 13 e Garrote et al. 14 relataram efeitos nulos. Esse feito pode ser devido ao consumo de uma dieta menos variada, portanto com menor fornecimento de nutrientes, pois o câncer oral parece estar relacionado com este tipo de dieta 13,20 .

Garrote et al. 14 relatando os resultados obtidos em Cuba, como parte de um estudo internacional conduzido sob a coordenação da IARC que utilizou questionário semelhante ao utilizado neste estudo, verificaram riscos aumentados, estatisticamente significativos, para o consumo de carnes e embutidos. Apesar das quantidades nos tercis de consumo terem sido semelhantes, esse mesmo efeito não foi observado em São Paulo, onde foram observadas associações inversas, porém não significativas estatisticamente. Tais achados parecem reforçar a tese de que, mais que 
os alimentos em si, a combinação dos mesmos, associados ao estilo de vida podem ser fatores determinantes na etiologia do câncer oral.

Entre os achados mais consistentes dos estudos epidemiológicos que investigaram o câncer oral e a dieta, estão os efeitos protetores das frutas e vegetais: praticamente todos os estudos relataram associações inversas 5,7,13,14,16,17,20,25. A mais alta categoria de freqüência de consumo mostra aproximadamente metade do risco. Neste estudo também evidenciou-se associação direta, porém não significativa estatisticamente. Os únicos resultados significativos em relação aos grupos de alimentos foram verificados para o consumo de cereais, com diminuição de $40 \%$ no risco para consumo no tercil intermediário e laticínios, aumento de $70 \%$ no risco para consumo maior que 11 porções semanais.

Estudos tipo caso-controle são em geral criticados por serem suscetíveis a vieses de seleção e informação. A esses problemas metodológicos adicionam-se outras dificuldades quando se investigam as relações entre câncer e dieta. Inicialmente, a inabilidade de se estimar com precisão e acurácia o consumo dietético. Há ainda as dificuldades para estabelecer ou recordar a verdadeira exposição. Outro problema é que as diferenças na ingestão de alimentos tendem a ser pequenas nos estudos em que os indivíduos são as unidades de medida. A magnitude da associação que pode ser observada é, portanto, muito menor e o poder para detectar as relações entre câncer e dieta é reduzido, independente do método de avaliação da dieta 24,26 .

Outra preocupação é o consumo calórico. É muito difícil estabelecer o papel do consumo calórico sobre a carcinogênese, já que, ao aumentar ou diminuir o mesmo, aumentam ou diminuem os outros constituintes da dieta que podem ser promotores ou protetores dos tumores 27 . Uma das abordagens mais utilizadas é fazer o ajuste pelas calorias pelo método dos resíduos 27 . No entanto, essa abordagem tem sido questionada, pois estudos recentes têm demonstrado que o peso dos indivíduos está mais relacionado com o dispêndio energético que com as estimativas de ingestão energética. Dessa forma, Day \& Ferrari 24 sugerem que se o objetivo é ajustar pelo consumo energético, então usar o peso como substituto pode ser melhor que ajustar pela energia per $s e$. Neste estudo, utilizamos como opção ao ajuste pelas calorias o ajuste simultâneo pelo peso relatado do indivíduo antes do início dos sintomas, e pelo número de porções consumidas.

Os hábitos alimentares, tais como a inclusão de frutas e verduras na dieta, podem refletir a posição sócio-econômica e nível educacional. As classes sociais inferiores tendem a ter uma dieta menos saudável 28. Giskes et al. 29 em inquérito populacional, relataram que adultos de menor renda consumiam uma menor variedade de frutas e vegetais que os de renda mais elevada. No Brasil, Pontes 30 relata maior consumo de leite e carnes nas famílias de classe de renda mais elevada. Por outro lado, educação e renda estão positivamente relacionadas. Parmenter et al. 31 relataram diferenças significativas em conhecimentos entre os grupos sociodemográficos, com homens tendo menor conhecimento que mulheres e o conhecimento declinando com o menor nível educativo e sócio-econômico. A instrução é provavelmente o critério mais utilizado no Brasil para separar as pessoas por nível sócio-econômico ${ }^{32}$. Neste estudo, o grau de instrução foi utilizado no modelo estatístico como variável de controle, para ajustar por estes possíveis efeitos de confusão.

Outras importantes variáveis são os hábitos de consumo de tabaco e álcool, os dois mais importantes e reconhecidos fatores de risco para o câncer oral. Dallongeville et al. 33 conduziram uma metanálise sobre a ingestão de nutrientes associados com tabagismo, e verificaram que fumantes tinham ingestão mais baixa de fibra, vitamina C, vitamina E e beta-caroteno, nutrientes correlacionados com ingestão de frutas e vegetais, ao lado de maior consumo de álcool e energia. O questionário utilizado na pesquisa permitiu um conhecimento detalhado da exposição ao tabaco e álcool, tanto em termos de período, duração e intensidade da exposição, sendo que as variáveis incorporadas no ajuste do modelo estatístico permitiram levar em conta os consumos médios apresentados pelos indivíduos participantes durante a vida.

As análises sugerem que o consumo regular, praticamente diário, do prato brasileiro de ricos e pobres: arroz e feijão, pode diminuir os riscos associados com o câncer oral, independente do consumo de álcool, tabaco, do grau de instrução e do local de nascimento. Essa mistura tradicional fornece proteína de bom valor biológico, com a vantagem de aportar fibras dietéticas, carboidratos complexos, ser baixa em gorduras saturadas e não possuir colesterol. Qual seria o exato mecanismo que pode responder ao efeito observado de proteção ao câncer oral, não é possível identificar a partir de um estudo com este delineamento. Não se pode deixar de aventar a possibilidade da ocorrência de uma associação espúria, por um fator ainda desconhecido, já que os considerados importantes foram controlados no desenho do estudo e na modelagem estatística. Tem-se relatado na literatura tendência ao menor consumo dos alimentos básicos, como o arroz e especialmente feijão 34 . Em compensa- 
ção, houve substancial aumento do consumo de proteínas, representadas pela carne bovina e de frango, em consonância com a transição nutricional 34,35 .

O estudo do qual foram retirados os dados deste trabalho é um dos maiores já realizados no Brasil para investigação do câncer oral até o momento, envolvendo centros em diferentes Estados da Federação. No entanto, o estudo não foi delineado especialmente para o estudo dos

\section{Resumo}

Neste estudo, investigou-se os fatores dietéticos associados com o câncer oral, em um estudo tipo caso-controle de base hospitalar no Município de São Paulo, Brasil, entre 1998 e 2002. Participaram 835 indivíduos, sendo 366 casos incidentes de câncer de cavidade oral ou faringe e 469 controles. Os dados de consumo alimentar foram obtidos por um Questionário de Freqüência Alimentar (QFA). Os valores de odds ratio (OR) e intervalos com 95\% de confiança (IC95\%) foram estimados por regressão logística não condicional, ajustada por potenciais fatores de confusão. Verificaram-se associações inversas entre o câncer oral e o consumo mais elevado de feijão, OR = 0,37 (IC95\%: 0,22-0,64), vegetais crus, OR =0,51 (IC95\%: 0,29-0,93) e para o tercil intermediário de arroz e massas, $\mathrm{OR}=0,56$ (IC95\%: $0,38-0,83$ ). Associações diretas foram observadas para o mais elevado consumo de ovos, OR = 1,84 (IC95\%: 1,23-2,75), batata, OR = 2,22 (IC95\%: 1,53-3,25) e leite, OR= 1,80 (IC95\%: 1,09-2,98). Alimentos básicos da dieta do brasileiro, como arroz e feijão, emergiram como fatores de proteção para o câncer oral, independente de outros fatores de risco.

Neoplasias Bucais; Dieta; Estudos de Casos e Controles fatores dietéticos. Apesar disso, foi possível identificar associações entre a dieta e o câncer oral, destacando-se o papel protetor de alimentos básicos da dieta brasileira, como o arroz e feijão. Se esse padrão é realmente favorável para a prevenção do câncer oral, é mais uma motivação para a reversão da tendência da adoção dos padrões dietéticos de sociedades afluentes, cujas conseqüências já reconhecidas são o aumento das doenças crônicas não transmissíveis.

\section{Colaboradores}

D. M. L. Marchioni participou de todas as etapas de desenvolvimento do artigo. R. M. Fisberg e M. R. D. O. Latorre participaram nas análises estatísticas, interpretação e discussão dos resultados do estudo. J. F. Góis Filho, L. P. Kowalski, M. B. Carvalho e M. Abrahão colaboraram na análise e revisão do texto. V. Wünch-Filho e J. Eluf Neto foram responsáveis pelo delineamento, condução e coordenação do estudo, tendo também colaborado na interpretação e discussão dos resultados.

\section{Agradecimentos}

O estudo foi desenvolvido com financiamento da Comissão Européia (IC18 CT970222) e da Fundação de Amparo à Pesquisa de São Paulo (processo 2001/17682). O presente trabalho foi realizado com o apoio da Coordenação para o Aperfeiçoamento de Pessoal de Nível Superior. 


\section{Referências}

1. Wünsch-Filho V. The epidemiology of oral and pharynx cancer in Brazil. Oral Oncol 2002; 38: 737-46.

2. Franceschi S, Bidoli E, Herrero R, Munoz N. Comparison of cancers of the oral cavity and pharynx worldwide: etiological clues. Oral Oncol 2000; 36:106-15.

3. Instituto Nacional de Câncer. Estimativa da incidência de câncer no Brasil 2006. http://www. inca.gov.br/estimativa2006 (acessado em 06/ Jan/2006).

4. Ferlay J, Bray F, Pisani P, Parkin DM. GLOBOCAN 2002: cancer incidence, mortality and prevalence worldwide. Lyon: IARC Press; 2004. (IARC Cancer Base, 5).

5. Stewart BW, Kleihues P. World cancer report. Lyon: IARC Press; 2003.

6. Kjaerheim K, Gaar M. Anderse A. The role of alcohol, tobacco and dietary factors in upper aerogastric tract cancer: a prospective study of 10,900 Norwegian men. Cancer Causes Control 1998; 9:99-108.

7. World Cancer Research Fund. Nutrition and the prevention of cancer: a global perspective. Washington DC: American Institute for Cancer Research; 1997.

8. Organização Mundial da Saúde. Classificação estatística internacional de doenças e problemas relacionados à saúde - 10a revisão. v. 1. São Paulo: Centro Colaborador da OMS para a Classificação de Doenças em Português; 1995.

9. International Agency for Research on Cancer. Tobacco smoking: monographs on the carcinogenic risk of chemical to humans. Lyon: IARC Press; 1986.

10. International Agency for Research on Cancer. Alcohol drinking: IARC monographs on the evaluation carcinogenic risk in humans. Lyon: IARC Press; 1988.

11. Marquart L, Jacobs Jr. DR, Slavin JL. Whole grains and health: an overview. J Am Coll Nutr 2000; 19 (3 Suppl):289S-90S.

12. Mathers JC. Pulses and carcinogenesis: potential for the prevention of colon, breast and other cancers. Br J Nutr 2002; 88 Suppl 3:S273-9.

13. Franceschi S, Favero A, Conti E, Talamini R, Volpe R, Negri E, et al. Food groups, oils and butter and cancer of the oral cavity and pharynx. Br J Cancer 1999; 80:614-20.

14. Garrote LF, Herrero R, Reyes RM, Vaccarella S, Anta JL, Ferbeye L, et al. Risk factors for cancer of the oral cavity and oro-pharynx in Cuba. Br J Cancer 2001; 85:46-54.

15. Petridou E, Zavras AI, Lefatzis D, Dessypris N, Laskaris G, Dokianakis G, et al. The role of diet and specific micronutrients in the etiology of oral carcinoma. Cancer 2002; 94:2981-8.

16. Kreimer AR, Randi G, Herrero R, Castellsague X, La Vecchia C, Franceschi S, et al. Diet and body mass, and oral and oropharyngeal squamous cell carcinomas:analysis from the IARC multinational casecontrol study. Int J Cancer 2006; 118:2293-7.
17. De Stefani E, Boffetta P, Ronco AL, Correa P, Oreggia F, Deneo-Pellegrini H, et al. Dietary patterns and risk of cancer of the oral cavity and pharynx in Uruguay. Nutr Cancer 2005; 51:132-9.

18. Chyou PH, Nomura AMY, Stemmermann GN. Diet, alcohol, smoking and cancer of the upper aerodigestive tract: a prospective study among Hawaii Japanese men. Int J Cancer 1995; 60:616-21.

19. Zheng T, Boyle P, Willet WC, Hu H, Dan J, Evstifeeva $\mathrm{TV}$, et al. A case-control study of oral cancer in Beijing, People's Republic of China. Associations with nutrient intakes, foods and food groups. Eur J Cancer B Oral Oncol 1993; 29B:45-55.

20. Levi F, Pasche C, La Vecchia C, Lucchini F, Franceschi S, Monnier P. Food groups and risk of oral and pharyngeal cancer. Int J Cancer 1998; 77:705-9.

21. Tavani A, Gallus S, La Vecchia C, Talamini, R Barbone F, Herreno R, et al. Diet and risk of oral and pharyngeal cancer. An Italian case control study. Eur J Cancer Prev 2001; 10:191-5.

22. Uzcudun AE, Retolaza IR, Fernandez PB, SanchezHernandez JJ, Grande AG, Garcia AG, et al. Nutrition and pharyngeal cancer: results from a casecontrol study in Spain. Head Neck 2002; 24:830-40.

23. Paiva SA, Russell RM. Beta-carotene and other carotenoids as antioxidants. J Am Coll Nutr 1999; 18:426-33.

24. Day NE, Ferrari P. Some methodological issues in nutritional epidemiology. In: Riboli E, Lamber R, editors. Nutrition and lifestyle: opportunities for cancer prevention. Lyon: IARC Press; 2002. p. 5-10. (IARC Scientific Publications, 156).

25. De Stefani E, Boffetta P, Oreggia F, Fierro L, Mendilaharsu M. Hard liquor drinking is associated with higher risk of cancer of the oral cavity and pharynx than wine drinking. A case-control study in Uruguay. Oral Oncol 1999; 34:99-104.

26. Willet WC. Diet and nutrition. In: Schottenfeld D, Fraumeni JF, editors. Cancer epidemiology and prevention. New York: Oxford University Press; 1996. p. 438-61.

27. Willet W, Stampfer M. Total energy intake: implications for epidemiologic analyses. Am J Epidemiol 1986; 124:17-27.

28. James WP, Nelson M, Ralph A, Leather S. Socioeconomic determinants of health: the contribution of nutrition to inequalities in health. BMJ 1997; 314:1545-9.

29. Giskes K, Turrell G, Patterson C, Newman B. Socio-economic differences in fruit and vegetable consumption among Australian adolescents and adults. Public Health Nutr 2002; 5:663-9.

30. Pontes NS. A informação de consumo alimentar: os dados da POF 1995-1996. In: Instituto Danone, organizador. Consumo alimentar: as grandes bases de informação. São Paulo: Instituto Danone; 2000. p. 47-51.

31. Parmenter K, Waller J, Wardle J. Demographic variation in nutrition knowledge in England. Health Educ Res 2000; 15:163-74.

32. Pereira MG. Epidemiologia: teoria e prática. Rio de Janeiro: Editora Guanabara Koogan; 2001. 
33. Dallongeville J, Marecaux N, Fruchart JC, Amouye P. Cigarrette smoking is associated with unhealthy patterns of nutrient intake: a meta-analysis. J Nutr 1998; 128:1450-7.

34. Levy-Costa RB, Sichieri R, Pontes NS, Monteiro CA. Disponibilidade domiciliar de alimentos no Brasil: distribuição e evolução (1974-2003). Rev Saúde Pública 2005; 39:530-40.
35. Monteiro CA, Mondini L, Costa RBL. Mudanças na composição e adequação nutricional da dieta familiar nas áreas metropolitanas do Brasil (19861996). Rev Saúde Pública 2000; 34:251-8.

Recebido em 16/Jan/2006

Versão final reapresentada em 31/Mai/2006

Aprovado em 10/Jul/2006 